\title{
Competencia lingüística del alumnado inmigrante en un contexto bilingüe ${ }^{1}$
}

\section{Linguistic competence of immigrant children in a bilingual context}

\author{
José Luis Navarro* y Ángel Huguet \\ Universidad de Lleida. Cataluña, España. \\ (Recepción: Septiembre 2007 - Aceptación: Octubre 2007)
}

\begin{abstract}
Resumen
Es bien conocida la relevancia que en los últimos años está teniendo el fenómeno de la inmigración en Cataluña (España). En consecuencia, nuestras escuelas se han transformado en centros donde la pluralidad étnica, religiosa, cultural y lingüística es cada día más evidente. Pero, a diferencia de otros territorios del Estado español, una de las características fundamentales de nuestro Sistema Educativo es su organización bajo los parámetros de la educación bilingüe. De esta manera, además de un profundo dominio del catalán, lengua propia de Cataluña y lengua usada como vehicular y de aprendizaje en la enseñanza, se persigue un buen conocimiento de la lengua castellana.

En este contexto, diferentes estudios llevados a cabo en países con larga tradición en la recepción de inmigrantes, así como los escasos trabajos desarrollados en Cataluña y en el Estado español, ponen en evidencia el papel clave del dominio de la lengua de la escuela tanto de cara a garantizar el éxito escolar como para la integración y la cohesión social.

Con este marco de fondo, nos situamos en el caso del Instituto de Educación Secundaria (IES) de Guissona, en la comarca de la Segarra (Cataluña), donde por circunstancias diversas el fenómeno migratorio ha sido muy importante a lo largo de la última década. El objetivo de nuestra investigación es el análisis de las competencias lingüísticas del alumnado de dicho centro, tomando como referencia el nivel de competencia lingüística de sus iguales autóctonos. En concreto, a partir de una muestra de 28 niños y niñas inmigrantes que fueron comparados con un grupo de 93 escolares autóctonos de su misma edad y nivel escolar, nuestro trabajo analiza el desarrollo de diferentes habilidades en lengua catalana y castellana.
\end{abstract}

Palabras clave: Catalán, castellano, competencia lingüística, alumnado inmigrante.

\begin{abstract}
It is a fact generally acknowledged that immigration has turned out to be a relevant phenomenon in Catalonia (Spain) for the last few years. As a consequence, our schools are a setting where ethnic, religious, cultural and linguistic plurality has become increasingly evident. However, in contrast to other territories of Spain, one of the fundamental characteristics of Catalonia's Educational System is that it is organised following the parameters of bilingual education. Thus, apart from promoting a high degree of competence in Catalan, which is both vehicular and language of instruction, a good knowledge of Castilian is also pursued.

In this context, different studies carried out in countries with a long tradition in the reception of immigrants, as well as the very few works developed in Catalonia and Spain, make evident the key role played by a good command of the language of the school. This is so both in terms of academic success as well as when dealing with integration and social cohesion.

Within this framework, this study focuses on the Secondary Education School of Guissona, in La Segarra district (Western Catalonia), where, for several reasons, the importance of the migratory phenomenon has grown progressively for the last decade. The aim of this paper is to present the analysis of the linguistic competence of immigrant students in this school, taking as a reference the level of linguistic competence of their autochthonous classmates. In short, our work analyses the development of different abilities in Catalan and Spanish in a sample of 28 immigrant boys and girls as compared to a group of 93 local students in their same educational level.

Key words: Catalan, Spanish, linguistic competence, immigrant children.
\end{abstract}

\footnotetext{
${ }^{1}$ El trabajo ha sido posible gracias a una ayuda de la Dirección General de Investigación del Ministerio de Educación y Ciencia (nº referencia SEJ2005-08944C02-02/EDUC), así como de la Generalitat de Catalunya - Agencia de Gestió d'Ajuts Universitaris i de Recerca ( $\mathrm{n}^{0}$ referencia 2006 ARAI 00011).

"Correspondencia a: José Luis Navarro, Ángel Huguet. Universitat de Lleida - Departament de Pedagogia i psicología, Complex de La Caparrella, s/n 25192 Lleida (España). Teléfono: +34 973702300 E-mail: jnavarro@pip.udl.es - huguet@pip.udl.es
} 


\section{Introducción}

Los procesos migratorios han introducido, entre otros aspectos, una nueva dimensión multilingüe y multicultural, tanto en la sociedad en general como en la educación formal en particular.

En este sentido, podemos constatar a partir de la revisión del padrón municipal de habitantes referido a 1 de enero de 2007 que en ese año había en España 4.482.568 extranjeros que representaban un 9,9\% de la población total (Instituto Nacional de Estadística, 2007). Por lo que se refiere a Cataluña, acogía 966.004 personas provenientes de otros países y ello representaba un 13,4\% de su población total. Estas cifras acentúan la singularidad del fenómeno de la inmigración en nuestro país si lo comparamos con el proceso seguido en otros Estados de nuestro entorno, especialmente a partir del año 2000.

Como es lógico, y a consecuencia de los procesos de reagrupamiento familiar, la escuela no ha sido ajena al cambio social experimentado por nuestro país. En este sentido, cada vez más, en escuelas e institutos del Estado español, estudiantes lingüística y culturalmente diversos constituyen una parte importante de la población escolar.

Así, según datos publicados recientemente (CIDE, 2006; Ministerio de Educación y Ciencia, 2007), de 57.406 alumnos extranjeros matriculados en enseñanzas no universitarias en el curso 1995/1996, se ha pasado a 529.461 en el 2005/2006, lo cual representa el 7,39\%. En el caso concreto de Cataluña supone, exactamente, el 10,2\%. Todo ello se refleja también en las comarcas leridanas. Así, al inicio del curso 2006/2007, el alumnado de origen inmigrante suponía el 14\% del total de 57.982 escolares matriculados en Educación Infantil, Primaria y Secundaria (Martí, 2006).

Esta realidad nos sitúa en un contexto de diversidad lingüística y cultural. Cataluña no es diferente y, a lo largo de la última década, la heterogeneidad identitaria, lingüística, cultural y étnica ha aumentado de manera importante Sin duda, eso se refleja en el sistema educativo que ha de afrontar problemas hasta hace poco desconocidos.

En este contexto enseñar a estos alumnos a dominar la lengua o lenguas de la sociedad donde viven, constituye uno de los objetivos centrales de la escolaridad. Consciente de este hecho, el propio profesorado suele resaltar lo determinante que resulta el dominio de la lengua que usa la escuela en el éxito o fracaso escolar de este alumnado y, en consecuencia, para conseguir esta finalidad se suelen dedicar múltiples esfuerzos; a pesar de todo ello, en numerosas ocasiones, los resultados obtenidos no responden ni a las expectativas ni al tiempo dedicado.

Es cierto que entre el profesorado existen ciertas creencias o ideas previas que condicionan las respuestas educativas. De esta manera, hay una tendencia a pensar que los niños inmigrantes escolarizados en los primeros cursos aprenden la lengua de una forma natural, por simple "inmersión" en nuestro medio escolar y sin necesidad de actividades ni programas específicos (Navarro y Huguet, 2006).

En el mismo sentido, estas ideas operan a niveles no estrictamente lingüísticos y se plasman de tal manera que se tiende a creer que en estos casos los escolares se adaptan rápidamente y de forma satisfactoria a las exigencias de nuestra escuela.

Probablemente, la diversidad de lenguas de origen y, de manera especial, los diferentes niveles de competencia en la lengua de instrucción de los recién llegados sea el elemento que genera una mayor preocupación en una escuela que tradicionalmente había optado por la promoción de la homogeneidad. En gran medida, a ello se suelen achacar gran parte de las dificultades que surgen en los procesos de enseñanza y aprendizaje con estos niños y niñas que, en definitiva, se traducen en un fracaso escolar muy superior al de sus pares autóctonos, y con mayor incidencia en los casos de incorporación tardía (Huguet y Navarro, 2006).

La situación descrita hasta aquí difiere de manera notable de las realidades que han suscitado muchas de las investigaciones y las reflexiones sobre la educación bilingüe. Así, de manera general, la enseñanza bilingüe se ha preocupado principalmente de organizar la educación para promover 


\section{JOSÉ LUIS NAVARRO Y ÁNGEL HUGUET}

conocimiento de una segunda lengua desde la escuela a grupos lingüísticamente homogéneos. Por eso, como mínimo es discutible realizar una transposición mecánica de algunos de los presupuestos de la educación bilingüe a la comprensión y la organización lingüística de nuestra realidad educativa (Vila, 2006).

Evidentemente, hablar sólo de los aspectos psicolingüísticos no es suficiente ya que hay otros factores que pueden estar incidiendo y que requieren también su abordaje de cara a una respuesta global a esta situación. Cuestiones como el enfoque comunicativo de la enseñanza de las lenguas, las actitudes hacia las lenguas de la escuela y la lengua propia, el marco organizativo de los centros, las interacciones en el marco del aula y del centro, etc., han de ser abordadas necesariamente. Es decir, procede una revisión de las actividades y planteamientos educativos del profesorado (Ruiz-Bikandi y Camps, 2007).

En este sentido, se han planteado propuestas sobre la enseñanza de las lenguas en la escuela, como puede ser el caso del currículum integrado, con el fin de superar dicha situación (Trujillo, 2007). No obstante, se han de entender como posibilidades en desarrollo que cabe investigar pero sobre las que no tenemos todavía suficiente evidencia empírica.

Pero, más allá del marco de la educación formal, cabe contemplar otro conjunto de factores sociales que no hemos desarrollado suficientemente en este artículo, por limitaciones obvias, pero que pueden ayudar a que este proceso se inserte en una perspectiva intercultural y de cohesión social, como es el caso del Plan Lengua y Cohesión Social que se está llevando a cabo en Cataluña (Besalú, 2006; Serra, 2006).

No obstante lo anteriormente expresado, el tema que nos ocupa en la investigación desarrollada es el que hace referencia a las competencias en la/s lengua/s de la escuela por parte del alumnado de origen inmigrante, ya que dicho conocimiento puede favorecer o no su proceso educativo.

Es cierto que la llegada de inmigrantes a nuestro territorio es relativamente reciente y, posiblemente como consecuencia de ello, son escasos los trabajos que analizan su integración en nuestro Sistema Educativo.

En función de ello, y en lo que sigue, nos referiremos a algunas investigaciones llevadas a cabo especialmente en el ámbito anglosajón y continuaremos con la exposición de otros estudios desarrollados en el Estado español.

Así, Cummins (2001), después de hacer una revisión de diversas investigaciones realizadas en el contexto de Estados Unidos (Collier, 19872; Thomas y Collier, 1997³), concluye:

\footnotetext{
${ }^{2}$ En dicho estudio se señala que los alumnos llegados a los Estados Unidos entre los 8 y los 12 años, con algunos años de escolarización en sus países de origen, precisan entre 5 y 7 años para alcanzar el nivel de sus pares angloamericanos en lectura, ciencias sociales y naturales. Aquéllos que llegan antes de los 8 años requieren entre 7 y 10 años para ello, y los que llegan después de los 12 años a menudo están ya fuera de la escuela antes de poder alcanzar dicho nivel.
}

\footnotetext{
${ }^{3}$ En este trabajo se revisaron más de 40.000 expedientes correspondientes a estudiantes que habían asistido durante al menos 4 años a las escuelas participantes en su investigación, escuelas que se habían agrupado en cinco grandes modelos de tratamiento de las lenguas y en las que se hallaban representadas más de 150 lenguas maternas (L1). Tras un análisis trasversal y longitudinal del progreso de los escolares, los autores concluyen que los estudiantes escolarizados en programas bilingües, cuyo conocimiento de la L1 es equivalente a su nivel de edad, suelen tardar entre 4 y 7 años en alcanzar un rendimiento promedio en inglés comparable al de sus iguales angloamericanos. Quienes fueron escolarizados en su país de origen y asisten a programas exclusivamente en L2 (inglés), pero mantienen un nivel en L1 equivalente a su nivel de edad, tardan entre 5 y 7 años. Los niños y niñas más pequeños que se escolarizan solo en L2 tardan entre 7 y 10 años en llegar a ese nivel, y muchos de ellos no lo alcanzan nunca a no ser que reciban apoyo en el hogar para su desarrollo académico y cognitivo.
} 
Los estudios de investigación desde principios de los años ochenta muestran que los estudiantes inmigrantes pueden adquirir rápidamente una fluidez considerable en la lengua dominante de la sociedad cuando están expuestos a la misma en el entorno y en la escuela. Sin embargo, pese a este rápido progreso en fluidez conversacional, generalmente hace falta un mínimo de cinco años (y a menudo muchos más) para recuperar el terreno con los hablantes nativos en los aspectos académicos de la lengua. (Cummins, 2001, p. 41)

Lo que dicho autor nos plantea es la enorme diferencia que existe en las habilidades implicadas en el uso del lenguaje en situaciones informales y en situaciones formales. Es decir, no es lo mismo ser capaz de hacer cosas con la lengua en un ámbito conversacional cara-a-cara, en el que aparecen un sinfín de señales no lingüísticas que permiten acceder al significado y al sentido de las producciones lingüísticas del interlocutor, que en un ámbito formal (por ejemplo, en actividades relacionadas con la lectura y la escritura) en las que el interlocutor no está presente y sólo se puede acceder a sus intenciones mediante procedimientos lingüísticos propios del locutor. En ambos casos las habilidades son muy distintas.

El mismo Cummins (2002) conceptualiza esta diferencia entre dichos niveles de uso del lenguaje como BICS (Basic Interpersonal Communicative Skills) y CALP (Cognitive Academic Language Proficiency).

Por lo que se refiere al Estado español, actualmente disponemos de una cierta base para pensar que las cosas no son muy diferentes en nuestro contexto respecto a lo visto hasta aquí con referencia a otros países con mayor tradición en los estudios sobre rendimiento escolar e inmigración.

En este sentido, tenemos que hacer referencia al trabajo de Navarro y Huguet (2005), en el que se revisan diferentes estudios. Entre los resultados de estas investigaciones cabe destacar que la mera asistencia a la clase ordinaria, cuando ésta se imparte en una lengua que no es la L1, no basta para alcanzar un dominio razonable de la L2, si no se establece paralelamente algún tipo de intervención educativa específica (Mesa y Sánchez, 1996). Dicho trabajo fue realizado en centros escolares de Melilla, y en él se analizaron muestras de alumnado entre los que había un alto porcentaje cuya L1 no era el castellano. La muestra de este estudio estaba formada por 285 escolares de Educación Infantil, $1^{\circ}$ y $3^{\circ}$ de Educación Primaria. Estos niños y niñas se hallaban escolarizados en tres colegios: uno de clase social media, otro de clase social media-baja y un tercero de clase social baja. De la muestra total, 166 alumnos eran de origen bereber y bilingües y el resto, 119, eran de origen europeo y monolingües.

Asimismo, las conclusiones de otro de los estudios (Díaz-Aguado, Baraja y Royo, 1996) ponían de manifiesto la existencia de dos niveles en el ritmo de aprendizaje de la lengua: las habilidades básicas de comprensión y expresión se adquirían rápidamente (entre dos meses y medio y cuatro meses), pero las dificultades más frecuentes se observaban en el nivel necesario para poder seguir las explicaciones de la clase y expresarse con precisión (entre uno y tres años, respectivamente). Esta investigación se llevó a cabo en siete centros de Educación Primaria de Madrid a fin de conocer cómo se producía el aprendizaje del castellano como L2 por parte de los escolares de origen inmigrante.

Cabe destacar asimismo el trabajo de Serra (1997) que, en el marco de una investigación más amplia cuyo principal objetivo era el de evaluar el rendimiento lingüístico y académico de alumnos no-catalanoparlantes en $4^{\circ}$ de Primaria de nivel sociocultural bajo y que seguían un proceso de inmersión, analiza una submuestra de 31 alumnos de procedencia extracomunitaria. La evaluación mostró que éstos conseguían niveles de conocimiento lingüístico y matemático muy pobres.

Otro estudio relevante es el realizado por Siguan (1998), en el que se analizaron cuatro centros 
escolares de la ciudad de Madrid y cuatro de la provincia de Barcelona (todos públicos) que acogían a un número considerable de alumnos inmigrados. La muestra de escolares extranjeros era de 325 y sus procedencias eran: Marruecos, Iberoamérica, Asia y África central. Los datos indican que este alumnado obtiene calificaciones más bajas que sus compañeros autóctonos en todas las asignaturas relacionadas directamente con la lengua. En dicho trabajo afirma lo siguiente:

La mayoría de los niños inmigrados en Cataluña y que comienzan la escolaridad antes de los seis años adquieren una competencia de las lenguas utilizadas en la escuela que les permite seguir las enseñanzas con aparente normalidad. La mayoría de ellos, sin embargo, presenta un déficit lingüístico, mayor o menor, en ambas lenguas, que afecta la calidad de sus adquisiciones escolares, un déficit que resulta más grave y que afecta fuertemente a los resultados escolares en el caso de los que se incorporan a la escuela más tarde (Siguan, 1998, pp. 114-115).

En un trabajo posterior, en 1999, el Grup de Recerca en Educació Especial de la Universitat de Girona (Fullana, Besalú y Vilà, 2003) analizó los resultados escolares de los alumnos hijos de inmigrantes extranjeros de origen marroquí y gambiano escolarizados en las comarcas de Girona. Dicho estudio se hizo con toda la población escolarizada en $6^{\circ}$ de Educación Primaria, $2^{\circ}$ y $4^{\circ}$ curso de la Educación Secundaria Obligatoria (ESO). Entre sus conclusiones cabe destacar que las materias que generan más fracaso son las lenguas (tanto la catalana como la castellana y la extranjera) y las matemáticas.

Asimismo, tenemos que hacer referencia al trabajo de Maruny y Molina (2000). Dicho trabajo se realizó en la comarca del Baix Empordà (Girona) y en él se analizó el proceso de adquisición del catalán por parte de un grupo de escolares de origen marroquí escolarizados desde $3^{\circ}$ de Educación Primaria a $4^{\circ}$ de Educación Secundaria. La muestra, formada por 49 alumnos y alumnas, estaba dividida en tres grupos en función del tiempo de estancia en Cataluña (menos de 18 meses, 18 a 36 meses y más de 36 meses), garantizando dos escolares por cada grupo y nivel escolar, e intentando el equilibrio entre niños-niñas, así como en relación a su L1 (árabe-bereber) y a sus capacidades cognitivas.

Los resultados de la investigación permiten señalar que al menos son necesarios 3 años para desarrollar suficiente competencia conversacional en la lengua de la escuela, 5 años para mostrar una comprensión lectora aceptable y, todavía más tiempo en relación con el dominio de la escritura y otras habilidades que garantizan el éxito escolar (ningún sujeto de la muestra alcanzó un nivel adecuado).

Finalmente, haremos mención de un estudio realizado por Navarro y Huguet (2005) centrado explícitamente en el estudio de las habilidades lingüísticas adquiridas por los escolares inmigrantes.

Este último trabajo implicó a todos los centros educativos que en la provincia de Huesca escolarizaban alumnado inmigrante en $1^{\circ}$ de ESO. En concreto, el grupo de inmigrantes estaba formado por 49 escolares ( 9 africanos, mayormente del Magreb, 20 americanos, casi todos ellos sudamericanos, y 20 europeos, fundamentalmente de la Europa del Este), de los cuales 25 eran niños y 24 niñas. A todos ellos se les aplicó una prueba de conocimiento de la lengua castellana cuyos resultados fueron contrastados con los obtenidos por otro grupo de 44 alumnos y alumnas autóctonos provenientes de dos centros de la misma provincia de características similares.

Dicha prueba, realizada a partir de un trabajo de Bel, Serra y Vila (1991), analiza los siguientes aspectos: Comprensión Oral, Morfosintaxis, Ortografía, Comprensión Escrita, Expresión Escrita, Expresión Oral Léxico-Morfosintaxis, Expresión Oral Organización de la Información, Fonética, Lectura Corrección Lectora, Lectura Entonación. Al final se obtienen dos puntuaciones globales: PG1 y PG2. El primer índice aparece a partir de las cinco primeras subpruebas que son escritas, 
mientras que en el segundo intervienen la totalidad de las pruebas (las anteriores más el resto que son orales).

Entre los resultados más importantes del estudio queremos destacar lo siguiente: la comparación del conocimiento lingüístico de los escolares inmigrantes (considerados en su conjunto e incluyendo, por tanto, a los de origen hispanoparlante) respecto a los autóctonos mostró diferencias significativas a favor de los autóctonos en todas las áreas analizadas, lo que se tradujo en puntuaciones medias distantes en casi 20 puntos, a partir de una escala de 0 a 100, tanto en los índices PG1 $(58,260$ para los inmigrantes y 77,760 para los autóctonos) como PG2 (59,642 para los inmigrantes y 77,145 para los autóctonos).

A partir de aquí, el objetivo principal de nuestra investigación es estudiar el proceso de adquisición de las lenguas de la escuela ${ }^{4}$ considerando especialmente este tipo de contextos. En nuestro caso pretendemos un enfoque cuantitativo y centrado en un momento concreto de la escolaridad que recoja una representación de los diferentes colectivos de inmigrantes establecidos en nuestro país. Dicha investigación se ha realizado en el IES de Guissona.

\section{Método}

\section{Variables}

Por lo que respecta las variables que hemos empleado en la investigación, se han tenido presentes algunas de las que aparecen en estudios clásicos sobre educación bilingüe, así como otras que se han mostrado relevantes en el caso de estudios con alumnado inmigrante. De este modo, las variables controladas han sido:

- Competencia lingüística en lengua catalana y lengua castellana

- Condición de inmigrante

- Escolarización previa (hemos eliminado al alumnado no escolarizado en su país de origen)

- Área o territorio de origen del alumnado

- Condición lingüística familiar.

\section{Participantes}

La localidad de Guissona (Lleida) concentra un porcentaje significativo de alumnado inmigrante, que refleja claramente la nueva realidad demográfica. Para ilustrar lo que acabamos de acentuar, podemos decir que en los últimos diez años la población de Guissona ha pasado de 2.500 a 5.000 vecinos y, según el censo, el $40 \%$ de estas personas son de origen inmigrante (García, 2006).

Por lo que se refiere a la elección de la Educación Secundaria Obligatoria (ESO), se ha valorado la relevancia de haber acabado la Educación Primaria y haber cambiado de etapa, con todo lo que supone el cambio de contexto en el desarrollo de las competencias lingüísticas de este alumnado.

Dado el interés de esta situación en Educación Secundaria, así como el nuevo contexto de atención al alumnado inmigrante en Cataluña, nos hemos centrado en esta etapa educativa y, específicamente, en los dos cursos finales de cada uno de los ciclos ( $2^{\circ}$ y $4^{\mathrm{a}}$ de ESO) del IES de Guissona (Lleida).

El estudio se ha llevado a cabo sobre la totalidad del alumnado $(\mathrm{N}=121)$ de estos dos cursos, tanto autóctonos $(\mathrm{N}=93)$ como inmigrantes $(\mathrm{N}=28)$.

La muestra final del alumnado inmigrante quedó formada por 14 chicos y 14 chicas.

\footnotetext{
${ }^{4}$ En el caso de Cataluña, el catalán y el castellano.
} 
La distribución de dicha muestra, en función del territorio de origen y lengua familiar, es la que aparece en la Tabla 1.

Tabla 1: Características de la muestra (entre paréntesis número de sujetos).

\begin{tabular}{|l|l|}
\hline $\begin{array}{c}\mathbf{N}^{\mathbf{0}} \text { de alumnos en función de } \\
\text { la zona geográfica de origen }\end{array}$ & \multicolumn{1}{c|}{ Lengua familiar } \\
\hline África: 5 & Árabe (4), Wolof (1) \\
\hline América: 2 & Castellano (2) \\
\hline Europa: 21 & Rumano (8), Ucraniano (6), Búlgaro (5), Portugués (2) \\
\hline
\end{tabular}

Por otra parte, dado que para la prueba de conocimiento de catalán y castellano no disponíamos de baremos adecuados al nivel educativo en que se llevó a cabo nuestro estudio, se optó por tomar como referente a la totalidad del alumnado autóctono de los dos cursos $\left(2^{\circ}\right.$ y $4^{\circ}$ de ESO), que suponen 93 alumnos (52 chicos y 41 chicas).

\section{Instrumentos}

Básicamente, se utilizaron dos tipos de instrumentos. Mientras el primero de ellos nos facilitó el control de las variables independientes, el segundo nos permitió examinar las habilidades lingüísticas en catalán y castellano por parte de la muestra.

Para obtener la información referente a: Condición de inmigrante, Área o territorio de origen, Escolarización previa, Condición linguística familiar, se procedió a partir de un cuestionario utilizado en estudios similares (Navarro y Huguet, 2005).

Respecto a la evaluación del conocimiento lingüístico catalán y castellano, nos servimos también de una prueba elaborada a partir de los trabajos de Bel, Serra y Vila (1991). Dicha prueba analiza los siguientes aspectos: Comprensión Oral (CO), Morfosintaxis (MS), Ortografía, (ORT), Comprensión Escrita (CE), Expresión Escrita (EE), Expresión Oral Léxico-Morfosintaxis (LMS), Expresión Oral Organización de la Información (OI), Fonética (FON), Lectura Corrección Lectora (LECT-C) y Lectura Entonación (LECT-E). Todos los apartados reciben una puntuación que oscila entre 0 y 100 puntos en función del número de aciertos y errores.

En su aplicación, la prueba consta de dos partes: una colectiva, que se realiza con el apoyo de un cuaderno de respuestas y contiene todas las pruebas escritas (CO, MS, ORT, CE, EE), y otra individual, que corresponde a las pruebas orales (LMS, OI, FON, LECT-C, LECT-E) y se lleva a cabo con la ayuda del material específico de la parte oral.

Existe un tiempo limitado de aplicación para cada una de las subpruebas y, por lo que se refiere a las puntuaciones, además de las propias de cada subprueba, se obtienen dos índices añadidos: PG1 que corresponde a la media de las cinco subpruebas escritas, y PG2 que se deriva de la media de las cinco subpruebas orales y de las cinco escritas.

Aunque la estructura es la misma, tanto para la lengua catalana como para la castellana, son pruebas diferentes por lo que se refiere a los textos, vocabulario, etc.

El tiempo empleado para la parte colectiva e individual fue de 75 y de 20 minutos, respectivamente, en cada una de las dos lenguas.

\section{Procedimiento}

El conjunto de los instrumentos descritos en el apartado anterior, se aplicó durante el último trimestre del curso escolar 2005/2006. Previamente, se contactó con las autoridades provinciales de educación y con el centro escolar implicado al objeto de clarificar las razones del estudio. Se establecieron los días en que tendrían lugar las entrevistas y pasaciones de pruebas, y se mantuvo una reunión informativa con el equipo directivo de cara a solicitar su colaboración.

Todos los escolares de origen inmigrante fueron entrevistados individualmente y, cuando apare- 
cieron dudas que resultaban claves, se solicitó la información a sus familias.

Aunque en este centro educativo, tanto en $2^{\circ}$ como en $4^{\circ}$ de ESO, hay tres clases por nivel, se hicieron dos grupos para la ejecución de la parte colectiva de la prueba. La parte individual se aplicó de forma aislada a la totalidad de escolares inmigrantes y al 25\% de los autóctonos, debido al tiempo necesario para la pasación a la muestra de dicho alumnado.

El personal responsable de la aplicación y corrección de protocolos fue especialmente entrenado a tal efecto.

\section{Tratamiento estadístico}

La obtención de datos estadísticos se realizó con ayuda del paquete integrado StatView for Windows v. 5.0.1. Se emplearon técnicas estadísticas descriptivas, el ANOVA y la prueba de comparación de medias de Fisher.

Mientras el ANOVA nos ha permitido comprobar los efectos de una o más variables independientes en la explicación de las diferencias sobre cada una de las variables dependientes, la prueba de Fisher nos ayudó a concretar las diferencias cuando el resultado del análisis de varianza se mostraba significativo. En cualquier caso el nivel de significación utilizado fue del 0.05.

\section{Resultados}

Comenzaremos este apartado presentando comparativamente los datos globales correspondientes al conocimiento de catalán y castellano obtenidos por autóctonos e inmigrantes.

En este análisis, dado que se utilizó la misma prueba en los dos cursos, y por razón de las dimensiones del grupo, se incluyen conjuntamente $2^{\circ}$ y $4^{\circ}$ de ESO.

Conocimiento de la lengua catalana: Alumnado autóctono vs. alumnado inmigrante

Para abordar esta cuestión, se procedió a partir de un análisis de la varianza contrastando los resultados de autóctonos e inmigrantes en cada una de las subpruebas que conforman la prueba de conocimiento de catalán, así como con relación a los índices PG1 y PG2.

En la Tabla 2 se presentan dichos datos, así como su nivel de significación.

Tabla 2: Comparación de las puntuaciones medias, en catalán, obtenidas por inmigrantes y autóctonos en las subpruebas CO, MS, ORT, CE, EE, LMS, OI, FON, LECT-C y LECTE, así como en los índices PG1 y PG2, y nivel de significación

\begin{tabular}{|c|c|c|c|c|c|}
\cline { 2 - 6 } \multicolumn{1}{c|}{} & \multicolumn{2}{c|}{ Inmigrantes } & \multicolumn{2}{c|}{ Autóctonos } & P \\
\cline { 2 - 6 } \multicolumn{1}{c|}{} & Media & $\sigma$ & Media & $\sigma$ & \\
\hline CO & 48,096 & 21,238 & 78,810 & 12,129 & $<0,0001^{*}$ \\
\hline MS & 31,657 & 24,934 & 74,453 & 16,177 & $<0,0001^{*}$ \\
\hline ORT & 64,657 & 22,186 & 85,437 & 13,797 & $<0,0001^{*}$ \\
\hline CE & 46,218 & 19,025 & 78,463 & 13,336 & $<0,0001^{*}$ \\
\hline EE & 71,036 & 31,113 & 89,933 & 6,186 & $0,0019^{*}$ \\
\hline PG1 & $\mathbf{5 2 , 3 3 3}$ & $\mathbf{2 0 , 1 8 1}$ & $\mathbf{8 1 , 4 1 9}$ & $\mathbf{9 , 3 6 8}$ & $<0,0001^{*}$ \\
\hline LMS & 65,340 & 17,665 & 86,287 & 8,801 & $<0,0001^{*}$ \\
\hline OI & 33,926 & 13,968 & 47,181 & 15,784 & $0,0013^{*}$ \\
\hline
\end{tabular}




\begin{tabular}{|c|c|c|c|c|c|}
\hline FON & 80,389 & 9,861 & 91,640 & 7,791 & $<0,0001^{*}$ \\
\hline LECT-C & 42,321 & 26,614 & 86,000 & 11,774 & $<0,0001^{*}$ \\
\hline LECT-E & 45,714 & 32,821 & 81,833 & 18,312 & $<0,0001^{*}$ \\
\hline PG2 & $\mathbf{5 2 , 9 3 6}$ & $\mathbf{1 6 , 5 4 7}$ & $\mathbf{8 0 , 0 0 4}$ & $\mathbf{8 , 0 9 4}$ & $<0,0001^{*}$ \\
\hline
\end{tabular}

Como se puede observar, las puntuaciones son notablemente inferiores en el caso del alumnado inmigrante para todas las subpruebas e índices. Además, los contrastes resultan significativos en todos los casos.

Evidentemente, ello se traduce en los índices derivados donde obtenemos valores de $\mathrm{F}_{1,56}=50,672$ $(\mathrm{p}=<0,0001)$ para PG1 y de $\mathrm{F}_{1,56}=63,947(\mathrm{p}=<0,0001)$ para PG2, que nos muestran un conocimiento sensiblemente menor de la lengua que vehicula los contenidos escolares en el alumnado inmigrante comparado con el alumnado autóctono.

Conocimiento de la lengua castellana: Alumnado autóctono vs. alumnado inmigrante

Se procedió de la misma forma y los resultados en relación a la lengua castellana mantienen la misma tendencia que con la lengua catalana, es decir, las puntuaciones son notablemente inferiores en el caso del alumnado inmigrante para todas las subpruebas e índices. Además, los contrastes resultan significativos en todos los casos, excepto en Lectura Entonación (LECT-E).

En la Tabla 3 se presentan dichos datos, así como su nivel de significación.

Tabla 3: Comparación de las puntuaciones medias, en castellano, obtenidas por inmigrantes y autóctonos en las subpruebas CO, MS, ORT, CE, EE, LMS, OI, FON, LECT-C y LECT-E, así como en los índices PG1 y PG2, y nivel de significación

\begin{tabular}{|c|c|c|c|c|c|}
\cline { 2 - 6 } \multicolumn{1}{c|}{} & \multicolumn{2}{c|}{ Inmigrantes } & \multicolumn{2}{c|}{ Autóctonos } & P \\
\cline { 2 - 6 } \multicolumn{1}{c|}{} & Media & $\sigma$ & Media & $\sigma$ & \\
\hline CO & 33,025 & 21,524 & 67,990 & 15,700 & $<0,0001^{*}$ \\
\hline MS & 36,150 & 24,448 & 72,572 & 18,944 & $<0,0001^{*}$ \\
\hline ORT & 59,529 & 28,600 & 80,173 & 19,389 & $0,0020^{*}$ \\
\hline CE & 40,814 & 22,084 & 69,913 & 16,341 & $<0,0001^{*}$ \\
\hline EE & 46,571 & 22,148 & 71,007 & 12,379 & $0,0019^{*}$ \\
\hline PG1 & $\mathbf{4 3 , 2 1 8}$ & $\mathbf{2 0 , 8 4 1}$ & $\mathbf{7 2 , 3 3 1}$ & $\mathbf{1 2 , 4 5 1}$ & $<0,0001^{*}$ \\
\hline LMS & 61,202 & 19,124 & 75,075 & 9,924 & $0,0009^{*}$ \\
\hline OI & 35,715 & 29,990 & 59,443 & 30,224 & $0,0040^{*}$ \\
\hline FON & 66,161 & 17,814 & 85,660 & 9,827 & $<0,0001^{*}$ \\
\hline LECT-C & 55,000 & 25,531 & 80,333 & 18,286 & $<0,0001^{*}$ \\
\hline LECT-E & 61,393 & 25,429 & 69,967 & 15,233 & $0,1221^{*}$ \\
\hline PG2 & $\mathbf{4 9 , 5 5 6}$ & $\mathbf{1 8 , 8 0 5}$ & $\mathbf{7 3 , 2 1 3}$ & $\mathbf{1 0 , 4 9 4}$ & $<0,0001^{*}$ \\
\hline
\end{tabular}

Evidentemente, ello se traduce en los índices derivados donde obtenemos valores de $\mathrm{F}_{1,56}=50,445$ ( $\mathrm{p}=<0,0001)$ para PG1 y de $\mathrm{F}_{1,56}=35,626(\mathrm{p}=<0,0001)$ para $\mathrm{PG} 2$, que nos muestran un conocimiento sensiblemente menor de la lengua castellana por parte del alumnado inmigrante comparado con el alumnado autóctono.

\section{Discusión}

Hemos podido constatar en nuestro estudio que el conocimiento lingüístico del alumnado inmigrante es inferior al del alumnado autóctono, tanto en catalán como en castellano, siendo estas diferencias estadísticamente significativas. Es de destacar que estos datos se hallan en la línea de 
las conclusiones planteadas en los estudios previos, tanto en el ámbito anglosajón (Cummins, 2001) como en el contexto español (Navarro y Huguet, 2005). Evidentemente, estas dificultades en el uso del lenguaje condicionan los resultados inferiores en el conjunto de aprendizajes y contenidos escolares y se traducen en un fracaso escolar muy superior al de sus pares autóctonos (Huguet y Navarro, 2006; Vila, 2006).

Asimismo, pueden sorprender estos datos cuando se piensa en la posible extrapolación de los programas de inmersión lingüística a este grupo de alumnos, pero no debemos olvidar que las condiciones que se dieron en la aplicación de los mismos, como es el caso de Cataluña, no son las que hay actualmente en contextos de inmigración.

Es decir, aspectos como el desconocimiento de la L1 por parte del profesorado, imposible, por otra parte, aun teniendo la mejor voluntad posible y dado el mapa lingüístico en el conjunto de los centros educativos, pueden conducir a ciertas prácticas educativas que actúen de forma negativa. Así, el proponer actividades a este alumnado pensando que es capaz de seguir ciertas tareas en las que no se da una contextualización del lenguaje y en las que no hay una negociación de los significados, puede tener la consecuencia de no desarrollar competencia ni en las lenguas de la escuela, con las implicaciones que eso conlleva en el proceso de los aprendizajes curriculares vehiculados a través de las mismas (Cummins, 2002).

En estas condiciones, el profesorado no debería esperar resultados milagrosos por parte del alumnado que está aprendiendo estas lenguas en el contexto escolar.

Además, funciona la creencia de que cuando un niño tiene un cierto dominio de la lengua en situaciones comunicativas habituales, ya es competente en todos sus ámbitos. Hay que clarificar, por tanto, esas diferencias entre un lenguaje conversacional que el niño puede adquirir pronto y el lenguaje académico, más formal y descontextualizado (Cummins, 2002). De lo contrario, se pueden generar excesivas expectativas que no se cumplen y que generan actitudes de desconcierto entre el profesorado.

Evidentemente, hablar sólo de los aspectos psicolingüísticos no es suficiente ya que hay otros factores que pueden estar incidiendo y que requieren también su abordaje de cara a una respuesta global a esta situación. Cuestiones como el enfoque comunicativo de la enseñanza de las lenguas, las actitudes hacia las lenguas de la escuela y la lengua propia, el marco organizativo de los centros, las interacciones en el marco del aula y del centro, etc., han de ser abordadas necesariamente.

Asimismo, hay que entender la actual situación de heterogeneidad en las aulas, desde el punto de vista lingüístico, cultural, etc., que no coincide con el marco de grupos lingüísticamente homogéneos, hecho éste considerado como valioso anteriormente para la práctica educativa y organización escolar (Vila, 2006). Todo ello requiere nuevas formas de abordar esta diversidad en las que la lengua sirva de instrumento para regular intercambios sociales.

Y como se ha planteado anteriormente, y más allá del marco cotidiano del día a día en el aula, cabe contemplar una función social de la educación en la que se incorporen perspectivas y elementos interculturales y de cohesión social que impliquen todo el tejido de la comunidad, entendida en sentido amplio (Besalú, 2006; Serra, 2006). En este sentido, y aunque no es el objetivo de este artículo, habrá que revisar y evaluar las aportaciones realizadas desde propuestas como el Plan de Lengua y Cohesión Social que se está llevando a cabo en Cataluña.

Pero volviendo a los elementos aportados por el estudio aquí presentado, y como puede apreciarse en la exposición previa, nuestra muestra es reducida y no podemos aventurarnos más allá de lo que nos permiten nuestros datos. En consecuencia, creemos necesario realizar estudios más amplios para verificar estos resultados y, por tanto, llevar a cabo una investigación más profunda 


\section{JOSÉ LUIS NAVARRO Y ÁNGEL HUGUET}

sobre el tema y con muestras más amplias que nos permitan analizar el papel de otras variables no contempladas en este estudio.

\section{Referencias}

Bel, A., Serra, J. M. y Vila, I. (1991). El coneixement de llengua catalana i castellana en acabar l'ensenyament obligatori el 1990. Documento no publicado. Barcelona: Departament d'Ensenyament-SEDEC.

Besalú, X. (2006). El Plan para la Lengua y la Cohesión Social (Plan LIC) del Gobierno de Cataluña. Revista Interuniversitaria de Formación del Profesorado, 20 (2), 45-68.

CIDE. (2006). El alumnado extranjero en el sistema educativo español (1995-2006). Boletín CIDE de temas educativos, 15.

Collier, V. P. (1987). Age and rate of acquisition of second language for academic purposes. Tesol Quaterly, 21, 617-641.

Cummins, J. (2001). ¿Qué sabemos de la educación bilingüe? Perspectivas psicolingüísticas y sociológicas. Revista de Educación, 326, 37-61.

Cummins, J. (2002). Lenguaje, poder y pedagogía. Madrid: Morata.

Díaz-Aguado, M. J., Baraja, A. y Royo, P. (1996). Estudio sobre la integración escolar de los alumnos extranjeros y el aprendizaje del castellano como segunda lengua. En M. J. Díaz-Aguado. Escuela y tolerancia (pp. 101-154). Madrid. Pirámide.

Fullana, J., Besalú, X. y Vilà, M. (2003). Alumnes d'origen África a l'escola. Girona: CCG Edicions.

García, F. (2006, octubre 8). Una población que no crece, se multiplica. Segre

Huguet, Á. y Navarro, J. L. (2006). Inmigración y resultados escolares: lo que dice la investigación. Cultura y Educación, 18 (2), 117-126.

Instituto Nacional de Estadística (2007). Padrón municipal. [en línea] http://www.ine.es [Consulta: 10 junio 2007]

Maruny, Ll. y Molina, M. (2000). Adquisició del català i competència comunicativa en alumnes d'origen marroquí a l'ensenyament obligatori. Memoria de investigación no publicada. Barcelona: Fundació Jaume Bofill.

Mesa, M. C. y Sánchez, S. (1996). Educación y situaciones bilingües en contextos multiculturales. Estudio de un caso: Melilla. Madrid: CIDE.

Ministerio de Educación y Ciencia (2007). Datos básicos de la educación en España en el curso 2006/2007. Madrid: MEC.

Martí, J. (2006, octubre 8). Los alumnos inmigrantes ya son amplia mayoría en media docena de colegios. Segre.

Navarro, J.L. y Huguet, A. (2005). El conocimiento de la lengua castellana en alumnado emigrante de $1^{\circ}$ de ESO. Un estudio empírico. Madrid: CIDE.

Navarro, J.L. y Huguet, A. (2006). Acerca de la competencia lingüística del alumnado de origen inmigrante, en Educación Secundaria. Revista Interuniversitaria de Formación del Profesorado, 20 (2), 69-80.

Ruiz-Bikandi, U. y Camps, A. (2007). La formación de docentes para situaciones plurilingües. Cultura y Educación, 19 (2), 101-103.

Serra, J. M. (1997). Inmersió lingüística, rendiment acadèmic y classe social. Barcelona: Horsori. 
Serra, J.M. (2006). El plan de lengua y cohesión social en Cataluña. Primeros datos de una investigación. Cultura y Educación, 18 (2), 159-172.

Siguan, M. (1998). La escuela y los inmigrantes. Barcelona: Paidós.

Thomas, W. P. y Collier, V. (1997). School effectiveness for language minority students. Washington. DC: National Clearinghouse for Bilingual Education.

Trujillo, F. (2007). Enseñar nuevas lenguas en la escuela: L1, L2, LE, NL. Revista de Educación, $343,71-91$.

Vila, I. (2006). Lenguaje, escuela e inmigración. Cultura y Educación, 18 (2): 127-142. 